\title{
Establishing Approaches and Practices Used by Midwives in Mental Health Assessment of Pregnant Women Making Visits to Antenatal Clinics in Public Health Facilities in Tharaka Nithi County
}

\author{
Kawira DM¹, Gitonga LK², Mukhwana ES ${ }^{3}$ \\ 1,2,3 Department of Nursing, Chuka University, P.O Box 109-60400, Chuka, Kenya \\ Corresponding Author: Kawira DM
}

\begin{abstract}
Mental health assessment is a critical practice to promote quality health among pregnant women and unborn children. The approaches and practices that midwives adopt at the antenatal clinics determined the mental health assessment success and the effectiveness of the subsequent treatment. Different studies concerning maternal mental health assessment have focused on issues of prevalence, barriers, and factors hindering or promoting assessment. However, there are limited studies exploring approaches and practices that enhance mental health screening. A sample of 45 midwives and 85 pregnant women was selected from public health facilities in TNC. Analysis of data and coding was done using SPSS with results presented in tables, bar graphs, and pie charts. Only 14\% of midwives used training as an approach towards promoting mental health. Both midwives and pregnant women registered low involvement in providing information. The county government and the hospitals' stakeholders should invest in approaches and practices that prioritize mental health assessment to prevent any mental health complications among pregnant women.
\end{abstract}

Keywords: Maternal mental health; mental health assessment, pregnant women, midwives, approaches and practices

\section{BACKGROUND}

Mental health is a global concern given the prevalence of mortality and morbidity associated with mental illnesses. Statistics indicate that pregnant women are among populations that report the highest prevalence estimates in mental health disorders. There are about $17 \%$ and $16 \%$ of pregnant women globally estimated to have mild and major mental disorders, respectively.[1] About $15 \%$ and 23\% of pregnant women are diagnosed with nervousness and anxiety disorders, respectively [2]. A study conducted to assess depression levels among pregnant women in Toronto, Canada reported the first onset of severe depression during the postnatal period [3]. The study showed that women reported thrice the odds of being diagnosed with severe depression during the postnatal period compared to other periods. Studies reveal that there is a huge gap in mental health assessment among women attending antenatal clinics during pregnancy. Whooley questions is one of the common approaches employed by midwives to assess pregnant women's mental wellbeing [4]. A survey conducted to assess the effectiveness of Whooley questions in mental health assessment showed that the approach was limited in covering a wide range of mental health issues surrounding pregnant women [5]. The survey failed to include vulnerable women who experience a variety of mental 
Kawira DM et.al. Establishing approaches and practices used by midwives in mental health assessment of pregnant women making visits to antenatal clinics in public health facilities in Tharaka Nithi County.

health problems likely to inform an explorative discussion on mental assessment.

Mental health screening is fundamental in promoting the quality mental health of pregnant women globally. There is accumulating evidence supporting the importance of maternal mental health screening and assessment to promote quality life among mothers and their children [6]. Studies have continued to explore designs and screening programs that promote quality mental health within the primary health care settings through improved screening and assessment while helping deal with the stigmatization of mental health among pregnant women. However, the maternal mental health prevalence reflects a concern in the employability of the practices and approaches used in mental health assessment. The prevalence of maternal postnatal depression varies from country to country with Australia reporting between $12 \%$ to $15 \%$ annually [7]. A significant percentage of these variations being explained by the approaches and processes used in mental health assessment.

Studies have explored different approaches and practices employed by midwives to improve the mental health wellbeing of pregnant women and enhance maternal health. For instance, [8] compared the impact of diagnostic codes and natural language processing (NLP) to screen suicidal-related behaviors among pregnant women and provide the most appropriate interventions. Electronic medical records helped improve the mental health screening sensitivity on suicidal behavior. There was a $76 \%$ performance improvement of the diagnostic codes compared to $36 \%$ performance of NLP. Electronic medical records continue to provide significant opportunities in developing countries to identify mental health problems early enough and provide appropriate interventions. Though the diagnostic code's performance exceeded that of NLP, the study recommended the integration of the two for improved screening sensitivity. [8]
Stated that the limitations of both approaches can be overcome through integration.

Third-world countries have a huge gap in addressing mental health concerns among pregnant women. According to [9], promoting quality mental health among pregnant women is a demanding and complex issue that requires multidisciplinary response through integrated approaches. A review of some of the available mental health screening tools among family physicians found out that clinical discussion was among the most employed approach for assessment [9]. Lack of standardized guidelines for different screening tools was a major concern identified in the study. A review of different studies established that most women from low-income backgrounds relied on selfrecognition to identify mental health problems because of financial challenges to assess clinical screening [10]. Kenya is a country whose women face many challenges associated with mental health assessment because of its economic status which adversely impacts the healthcare system.

Currently, Kenya employs different screening tools for diagnosis and treatment plans among mentally ill patients. The Patient Health Questionnaire-9 item (PHQ9) is commonly used in most health facilities. PHQ-9 is easily administered and extensively in screening mental health problems among pregnant women [11]. Another tool that is used for mental health assessment among pregnant and postpartum women in Kenya is the Edinburgh Postnatal Depression Scale (EPDS) [12]. However, the PHQ-9 is preferred in most healthcare settings because of its applicability in the general population, primary care, and reproductive health. Other studies have insisted that EPDS and PHQ-9 are westernized screening methods and lack the cross-cultural equivalence and adequate precision when applied in developing countries [13]. As a result, [13] conducted a pilot study to assess the impact of locally 
Kawira DM et.al. Establishing approaches and practices used by midwives in mental health assessment of pregnant women making visits to antenatal clinics in public health facilities in Tharaka Nithi County.

perinatal depression approaches among pregnant women in Kiambu County, Kenya to assess their efficiency. The two approaches, Thinking Healthy, and Healthy Moms offered automated mental health assessments to pregnant women. The approaches reported increased women's interest in mental health assessment by dealing with stigmatization associated with a mental health assessment. This paper reports the findings of different approaches and practices that Tharaka Nithi County employs to assess the mental health of pregnant women attending public health facilities.

\section{METHOD}

A descriptive cross-sectional research design was employed to identify the approaches and practices that midwives in public health facilities in TNC employ for mental health assessment of pregnant women attending the ANC clinics. The choice of the study design owes its suitability in the analysis of nonquantifiable issues [14]. The design was also suitable for collecting data from the respondents at a specific point in time. The research design helped with data collection and reporting of results with minimal variable manipulation. The study was conducted at the public health facilities offering antenatal care services in TharakaNithi County. These facilities included Kibung'a hospital, Marimanti hospital, Magutuni, and Chuka County Referral Hospital. The four public health facilities are found in different administrative divisions of the county.

The study population comprised midwives working the four public health facilities offering ANC services and pregnant women attending clinics at those health facilities. There study targeted about 228 midwives and 850 pregnant women who formed the sampling frame. The appropriate sample size was informed by [15] recommendation of a study sample of $10 \%$ to $30 \%$ of the target population. The study sampled $10 \%$ of each group of respondents and ended up with a sample size of 85 pregnant women and 45 midwives. The latter were sampled purposively based on their availability while cluster sampling was used to obtain a representative sample from each of the public health facilities.

The study variables were collected using researcher-administered questionnaires with close-ended questions. The researcher preferred the close-ended questionnaires owing to their robustness in obtaining accurate information within a short period [16]. In-depth interviews helped find out the approaches that midwives used for mental screening. Respondents had the opportunity to choose where they wanted the in-depth interviews to be conducted to minimize any harm caused to them during the study. Piloting of the instruments was done at Chogoria hospital by interviewing 10 women and 4 midwives. The research instruments were validated through an item analysis to check for face and content validity. A re-test method was employed to check for the reliability of the research instruments. The Cronbach alpha reliability test produced a correlation coefficient of 0.91 which showed that the instruments' have a higher degree of consistency in producing similar results if the study was repeated.

Approval to conduct the study was obtained from National Commission for Science Technology and Innovation (NACOSTI) and the Chuka University Research Committee. The researcher obtained permission from the hospital administration before interviewing the midwives. Written informed consent was secured for each of the participants before engaging in the study.

Data was coded in Statistical Package for Social Sciences V.26 (SPSS) and then analysis was conducted using the same software. Descriptive statistics (percentages) were generated and a Chisquare test was performed to examine to maternal health assessment. Results were 
Kawira DM et.al. Establishing approaches and practices used by midwives in mental health assessment of pregnant women making visits to antenatal clinics in public health facilities in Tharaka Nithi County.

presented visually using bar graphs, pie charts, and frequency tables.

\section{RESULT}

Table 1: Socio-demographic factors

\begin{tabular}{|l|l|l|}
\hline Variable & Frequency & Percentage \\
\hline Gender & & \\
\hline Female & 30 & 66.7 \\
\hline Male & 15 & 33.3 \\
\hline Academic Qualifications & & \\
\hline Certificate & 3 & 7.5 \\
\hline Diploma & 28 & 62.2 \\
\hline Bachelor's Degree & 9 & 20.0 \\
\hline Master's Degree & 5 & 12.5 \\
\hline Working Experience & & \\
\hline $1-3$ years & 0 & 0 \\
\hline $4-6$ years & 3 & 6.7 \\
\hline $7-9$ years & 5 & 11.1 \\
\hline $10-12$ years & 28 & 62.2 \\
\hline Over 12 years & 9 & 20.0 \\
\hline Length of service & & \\
\hline $1-3$ years & 2 & 4.4 \\
\hline $4-6$ years & 3 & 6.7 \\
\hline $7-9$ years & 5 & 11.2 \\
\hline $10-12$ years & 26 & 57.7 \\
\hline Over 12 years & 9 & 20.0 \\
\hline
\end{tabular}

From Table 2, the study established that the number of female midwives (66.7\%) was twice their male counterparts (33.3\%). More than half of the midwives $(62.2 \%)$ had a diploma in nursing/midwifery while 20\% had a bachelor's degree. About $13 \%$ of them had a master's degree with $7.5 \%$ having a certificate qualification. A majority of the midwives (62.2\%) had 10 to 12 years of work experience with 20\% having more than 12 years experience as nurse/midwives. Approximately $18 \%$ had between 4 to 9 years of work experience. Similarly, almost $78 \%$ of the midwives had been working for more than 9 years with about $19 \%$ having worked for about 4 to 9 years. Those with 3 years of service and below were only $4.4 \%$. The results indicated a significant proportion of the midwives with over 7 years of service and work experience.

\section{Approaches and Practices on maternal mental health assessment}

Midwives' training was one of the approaches through which the public health facilities in TNC promoted mental health assessment for pregnant women. Figure 1 shows that $86 \%$ of the midwives had received training on mental health assessment with the remaining 14\% confirming that the county's ministry of health had not offered them any in-service training on mental health assessment for the expectant mothers.

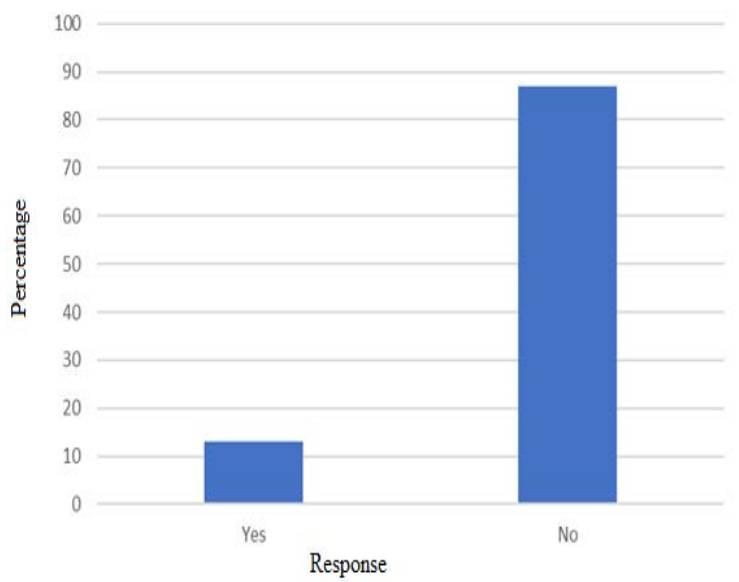

Figure 1: Proportion of Midwives trained on maternal mental health assessment in TNC's public health facilities

The study went further to identify major targets of mental health screening among the public health facilities. Results showed that a majority of the midwives (26.7\%) were concerned with identifying the main risks of mental health problems during the assessment (Table 2). As a result, they were more focused on identifying the most vulnerable women and giving them a priority in the treatment plan. Nearly $27 \%$ of the midwives were more concerned with the mitigation of maternal depression while $11.1 \%$ of the midwives focused more on the reduction of the number of women that did not require further assessment to give way to those that needed much attention.

Table 2: Target of Mental health screening among midwives

\begin{tabular}{|l|l|l|}
\hline Response & Frequency & Percentage \\
\hline $\begin{array}{l}\text { Reducing the number of women who } \\
\text { do not require further assessment }\end{array}$ & 5 & 11.1 \\
\hline $\begin{array}{l}\text { To highlight the women who are at } \\
\text { an increased risk }\end{array}$ & 28 & 62.2 \\
\hline $\begin{array}{l}\text { To mitigate the cases of maternal } \\
\text { depression }\end{array}$ & 12 & 26.7 \\
\hline Total & $\mathbf{4 5}$ & $\mathbf{1 0 0 . 0}$ \\
\hline
\end{tabular}

Results to establish the proportion of women requesting mental health screening at the public health facilities during their antenatal clinic showed that only $9 \%$ of the midwives had gotten requests from their 
Kawira DM et.al. Establishing approaches and practices used by midwives in mental health assessment of pregnant women making visits to antenatal clinics in public health facilities in Tharaka Nithi County.

patients on mental health screening (Figure 2). The remaining $91 \%$ of the midwives reported not having obtained any request for a mental health screening from the pregnant women attending the ANC.

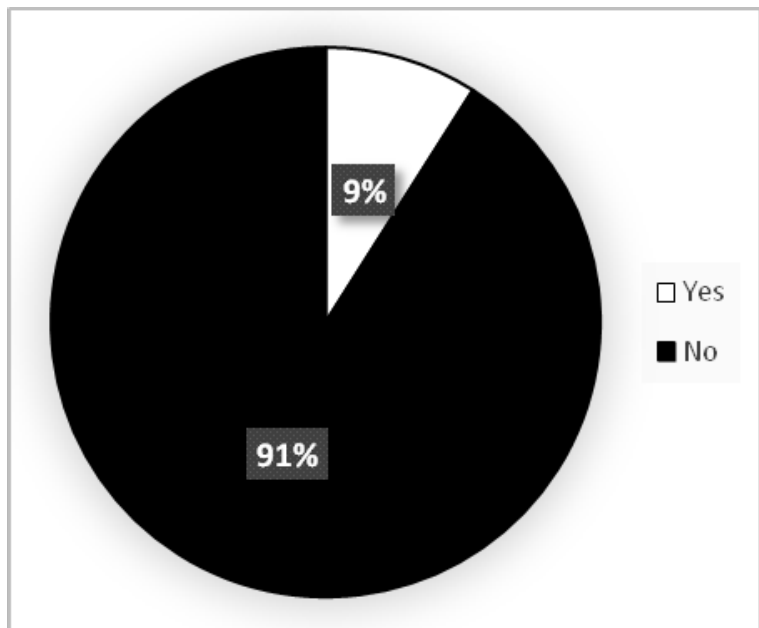

Figure 2: Request for Mental Health Screening

Further, the study sought to establish how often the public health facilities were involved in providing information about mental health screening to mothers attending the ANC clinics. A majority (68.9\%) of the midwives admitted to rarely educating the patients on the importance of mental health screening with only $8.9 \%$ of them addressing the issue (Figure 3). The remaining $22.2 \%$ indicated that they often informed their patients about the need to have a mental screening.

\begin{tabular}{|l|l|l|}
\hline Response & Frequency & Percentage \\
\hline Rarely & 31 & 68.9 \\
\hline Often & 10 & 22.2 \\
\hline Not sure & 0 & 0 \\
\hline Always & 4 & 8.9 \\
\hline Total & $\mathbf{4 5}$ & $\mathbf{1 0 0 . 0}$ \\
\hline
\end{tabular}

Figure 3: How often midwives gave mothers information on the importance of mental health screening

A Chi-square test to determine whether approaches and practices used in mental health assessment were effective on mental health screening among the pregnant women provided adequate evidence of the association between the two variables at a $5 \%$ level of significance $(\mathrm{p}<0.05)$ (Table 3$)$. The results showed that a significant relationship between the mental health assessment approaches and practices and mental health screening in the TNC's public health facilities.

Table 3: Chi-Square Test on Approaches and Practice used in Mental Health Assessment among the Pregnant Women and Effectiveness of Mental Health Assessment

\begin{tabular}{|l|l|l|l|l|l|}
\hline & Value & df & $\begin{array}{l}\text { Asymptotic Significance } \\
\text { (2 sided) }\end{array}$ & $\begin{array}{l}\text { Exact sig } \\
\text { (2-sided) }\end{array}$ & $\begin{array}{l}\text { Exact sig } \\
\text { (1-sided) }\end{array}$ \\
\hline Pearson Chi-square & $6.1124^{\mathrm{a}}$ & 1 & .001 & & \\
\hline Continuity correction & 6.0168 & 1 & .006 & & \\
\hline Likelihood ratio & 7.8614 & 1 & .004 & & \\
\hline Fishers exact test & & & & 0.07 & 0.10 \\
\hline Linear by linear association & 6.557 & 1 & .016 & & \\
\hline N of Valid cases & 45 & & & & \\
\hline
\end{tabular}

\section{DISCUSSION}

Hospitals and healthcare workers have a mandate by the public health to provide support to patients with mental problems by adopting approaches and practices that focus on the latter's wellbeing [17]. However, studies reveal that most nurses/midwives have failed to support their patients through mental health practice for lack of confidence [18]. Findings indicated that training on mental health assessment among the midwives was limited (14\%) with the county government contributed immensely to the lack of training. Findings on the reasons for mental health screening among pregnant women concurred with those of [19] on the need to promote early detection of mental health problems among pregnant women to save mothers' lives and that of the unborn children. The public health facilities targeted more on women with increased risk of mental problems and heightened mental health screening to mitigate the maternal depression among the affected. TNC's public health facilities understand the WHO's consideration of improved child and maternal health services by promoting mental health assessment. As noted by [20] mental health assessment enhances protection, prevention, and early 
Kawira DM et.al. Establishing approaches and practices used by midwives in mental health assessment of pregnant women making visits to antenatal clinics in public health facilities in Tharaka Nithi County.

identification of patients at high risks of mental ill-health and mortality.

There was a concern with the rate at which the pregnant women sought mental health screening services from the public health facilities. Similarly, the midwives were reluctant with providing information to pregnant women on the importance of mental health assessment. According to [21] effective mental health assessment depends on how much the health professionals educate patients on the need for screening and patients' willingness and awareness of the healthcare service. However, the findings indicated that both the midwives and the patients were reluctant about the mental health assessment leading to the low rates of screening. Limited involvement of midwives and patients in approaches and practices meant to promote mental health assessment in public health facilities in TNC poses a major challenge to prenatal care in the county.

\section{CONCLUSION}

Midwives training being one of the most fundamental approaches to enhance mental health assessment, there is limited training in public health facilities of Tharaka Nithi County. The county government of TNC has failed in providing the necessary support to promote training among the county's midwives. The county's ministry of health should invest in creating awareness among women on the importance of mental health screening for pregnant women. The study recommended the public health facilities invest in the implementation of mental health assessment approaches and practices because they are critical in promoting increased mental screening and wellbeing.

\section{Competing Interests}

Authors have declared that no competing interests exist.

\section{Acknowledgement: None}

Source of Funding: None

\section{Ethical Approval: Approved}

\section{REFERENCES}

1. Levis B, Negeri Z, Sun Y, Benedetti A, Thombs BD. Accuracy of the Edinburgh Postnatal Depression Scale (EPDS) for screening to detect major depression among pregnant and postpartum women: systematic review and meta-analysis of individual participant data. BMJ. 2020 Nov 11;371.

2. Milgrom J, Gemmill AW. Screening for perinatal depression. Best Practice \& Research Clinical Obstetrics \& Gynaecology. 2014 Jan 1;28(1):13-23.

3. Stewart DE, Robertson E, Dennis CL, Grace SL, Wallington T. Postpartum depression: Literature review of risk factors and interventions. Toronto: University Health Network Women's Health Program for Toronto Public Health. 2003 Oct:1-289.

4. Marsay C, Manderson L, Subramaney U. Validation of the Whooley questions for antenatal depression and anxiety among low-income women in urban South Africa. South African Journal of Psychiatry. 2017 Jan 1;23(1):1-7.

5. Alliance BF. Perinatal mental health: experiences of women and Health Professionals. London: Boots Family Trust. 2013.

6. Bayrampour $\mathrm{H}$, McNeil DA, Benzies K, Salmon C, Gelb K, Tough S. A qualitative inquiry on pregnant women's preferences for mental health screening. BMC pregnancy and childbirth. 2017 Dec;17(1):1-1.

7. Norhayati MN, Hazlina NN, Asrenee AR, Emilin WW. Magnitude and risk factors for postpartum symptoms: a literature review. Journal of affective Disorders. 2015 Apr 1;175:34-52.

8. Zhong QY, Karlson EW, Gelaye B, Finan S, Avillach P, Smoller JW, Cai T, Williams MA. Screening pregnant women for suicidal behavior in electronic medical records: diagnostic codes vs. clinical notes processed by natural language processing. BMC medical informatics and decision making. 2018 Dec;18(1):1-1.

9. Noonan M, Doody O, Jomeen J, O’Regan A, Galvin R. Family physicians perceived role in perinatal mental health: an integrative review. BMC family practice. 2018 Dec;19(1):1-22. 
Kawira DM et.al. Establishing approaches and practices used by midwives in mental health assessment of pregnant women making visits to antenatal clinics in public health facilities in Tharaka Nithi County.

10. Hansotte E, Payne SI, Babich SM. Positive postpartum depression screening practices and subsequent mental health treatment for low-income women in Western countries: a systematic literature review. Public Health Reviews. 2017 Dec;38(1):1-7.

11. Velloza J, Njoroge J, Ngure K, Thuo N, Kiptinness C, Momanyi R, Ayub S, Gakuo S, Mugo N, Simoni J, Heffron R. Cognitive testing of the PHQ-9 for depression screening among pregnant and postpartum women in Kenya. BMC psychiatry. 2020 Dec;20(1):1-4.

12. Khanlari S, Eastwood J, Barnett B, Naz S, Ogbo FA. Psychosocial and obstetric determinants of women signalling distress during Edinburgh Postnatal Depression Scale (EPDS) screening in Sydney, Australia. BMC pregnancy and childbirth. 2019 Dec;19(1):1-4.

13. Green EP, Tuli H, Kwobah E, Menya D, Chesire I, Schmidt C. Developing and validating a perinatal depression screening tool in Kenya blending Western criteria with local idioms: a mixed methods study. Journal of Affective Disorders. 2018 Mar 1;228:49-59.

14. Kothari CR. Research methodology: Methods and techniques. New Age International; 2004.

15. Orodho JA. Techniques of Writing Research Proposals and Reports in Education and Social Sciences. Bureau of Educational Research. Kenyatta University, Nairobi, Kenya: Kanezja HP Enterprises. 2008.
16. Desai SC, Reimers S. Comparing the use of open and closed questions for Web-based measures of the continued-influence effect. Behavior research methods. 2019 Jun;51(3):1426-40.

17. Crabbe K, Hemingway A. Public health and wellbeing: A matter for the midwife?. British Journal of Midwifery. 2014 Sep 2;22(9):634-40.

18. Jones M, Robson D, Whitfield S, Gray R. Does psychopharmacology training enhance the knowledge of mental health nurses who prescribe?. Journal of psychiatric and mental health nursing. 2010 Nov;17(9):80412.

19. Furber CM, Garrod D, Maloney E, Lovell $\mathrm{K}$, McGowan L. A qualitative study of mild to moderate psychological distress during pregnancy. International journal of nursing studies. 2009 May 1;46(5):669-77.

20. World Health Organization. Global tuberculosis control: epidemiology, strategy, financing: WHO report 2009. World Health Organization; 2009.

21. Bonas S, Ockleford E. Barriers to accessing mental health services for postnatal depression in the UK: a literature review.

How to cite this article: Kawira DM, Gitonga LK, Mukhwana ES. Establishing approaches and practices used by midwives in mental health assessment of pregnant women making visits to antenatal clinics in public health facilities in Tharaka Nithi County. Int J Health Sci Res. 2021; 11(12):9-15. DOI: https://doi.org/ 10.52403/ijhsr.20211202 